This item was submitted to Loughborough's Research Repository by the author.

Items in Figshare are protected by copyright, with all rights reserved, unless otherwise indicated.

\title{
Analysis of single hole simulated battle damage on a wing using particle image velocimetry
}

\section{PLEASE CITE THE PUBLISHED VERSION}

http://arc.aiaa.org/doi/abs/10.2514/6.2015-2573

\section{PUBLISHER}

(c) American Institute of Aeronautics and Astronautics

\section{VERSION}

AM (Accepted Manuscript)

\section{PUBLISHER STATEMENT}

This work is made available according to the conditions of the Creative Commons Attribution-NonCommercialNoDerivatives 4.0 International (CC BY-NC-ND 4.0) licence. Full details of this licence are available at: https://creativecommons.org/licenses/by-nc-nd/4.0/

\section{LICENCE}

CC BY-NC-ND 4.0

\section{REPOSITORY RECORD}

Almond, Mathew T., Peter M. Render, and Alastair Duncan Walker. 2015. "Analysis of Single Hole Simulated Battle Damage on a Wing Using Particle Image Velocimetry”. figshare. https://hdl.handle.net/2134/18186. 


\title{
Analysis of Single Hole Simulated Battle Damage on a Wing Using Particle Image Velocimetry
}

\author{
Mathew T. Almond ${ }^{*}$ Peter M. Render ${ }^{\dagger}$ A. Duncan Walker ${ }^{\ddagger}$ \\ Aeronautical \&3 Automotive Engineering Dept. Loughborough University, Loughborough, LE11 $3 T U$
}

\begin{abstract}
Particle Image Velocimetry (PIV) has been used to map the complex flow field generated by simulated battle damage to a two-dimensional wing. Previous studies have relied on surface flow visualisation techniques to study the flow but here PIV data has enabled the flow field away from the surface to be analysed for the first time. Damage was simulated by a single hole with a diameter equal to $20 \%$ of the chord, located at mid-chord. Wind tunnel tests were conducted at a Reynolds number of 500,000 over a range of incidences from $0-10^{\circ}$ with two-component PIV measurements made on three span-wise planes; on the damage centre line and offset by 0.5 and 1.0 hole radii. The PIV data was seen to be in good agreement with existing surface flow visualisation showing strong evidence of the formation of a horse shoe vortex, a counter-rotating vortex pair and reverse flow regions. Large variations in the flow structure were observed over the range of incidences tested as the jet transitioned from weak at lower angles to strong at higher angles. The data also revealed some significant differences in the flow compared to classic Jets In Cross-Flow (JICF) behaviour. Notably in the case of battle damage the jet never fully occupies the hole and jet velocity profile is highly skewed towards the rear of the hole. Additionally, the measured velocity ratios are much less than would be expected for typical JICF. For example, strong jet behaviour is observed at a velocity ratio as low as 0.22 whereas JICF studies would suggest a much higher ratio $(>2)$ is required. Increasing velocity ratio has been related to a reduction in lift and an increase in drag. At the highest incidence tested $\left(10^{\circ}\right)$ the velocity ratio of 0.32 resulted in a reduction of the lift coefficient by 0.18 and an increase in the drag coefficient of 0.035 .
\end{abstract}

\section{Nomenclature}

$\begin{array}{llll}C_{d} & \text { Drag Coefficient } & \mathrm{R} & \text { Radius of Hole } \\ C_{l} & \text { Lift Coefficient } & \text { CVP } & \text { Counter-Rotating Vortex Pair } \\ C_{p} & \text { Pressure Coefficient } & \text { HSV } & \text { Horse-Shoe Vortex } \\ D & \text { Diameter of Hole } & \text { JICF } & \text { Jets in Cross Flow } \\ d C_{d} & \text { Drag Coefficient Increment } & \text { Nd:YAG Neodymium-doped: Yttrium Aluminium } \\ d C_{l} & \text { Lift Coefficient Increment } & \text { Parnet } \\ d t & \text { Interframe Time } & \text { Particle Image Velocimetry } \\ J & \text { Momentum Flux Ratio } & & \\ L_{I} & \text { Linear Dimension of Interrogation Area } & G r e e k & \\ U & \text { Velocity } & \alpha & \text { Incidence Angle } \\ V_{R} & \text { Velocity Ratio }\left(U_{j e t} / U_{\infty}\right) & \Delta x & \text { Displacement Vector in Image Domain } \\ X & \text { X Co-ordinate } & \infty & \text { Freestream } \\ x / c & \text { Location along the wing chord } & \rho & \text { Density }\end{array}$

*PhD Research Student, Email: M.Almond@lboro.ac.uk.

†Senior Lecturer in Aircraft Aerodynamics, Email: P.M.Render@lboro.ac.uk.

‡Senior Lecturer in Applied Aerodynamics, Email: A.D.Walker@lboro.ac.uk 


\section{Introduction}

It is common for manned military aircraft, and increasingly Unmanned Aerial Vehicles (UAV's) to fly in areas where the risk of damage is high. Survivability is a key consideration when designing aircraft that fly in such places. Although structural integrity is very important, it is also important to consider the aerodynamic effects of battle damage to a wing. As far back as the 1960's, studies were being undertaken to determine the effects of battle damage on the aerodynamic capabilities of a wing or aircraft. In 1968, Hayes ${ }^{1}$ simulated wing damage on a swept wing model by removing wing panels. Although this early work was crude in its approach, it provided a basic understanding of the effects of battle damage. In the 1990's, Irwin ${ }^{2}$ developed a systematic and reliable technique to test battle damage by using simulated damage with uniform hole shapes and sizes in relation to chord length. As well as Irwin's work, investigations have been carried out into the effects of damage holes with different shapes (Mani and Render) ${ }^{3}$, damage from live fire (Robinson and Leishman $)^{4}$ and three dimensional tests using wings of finite span (Samaad-Suhaeb) ${ }^{5}$ and (Djellal $)^{6}$

When performing battle damage studies, simulated damage is commonly used. This technique uses regular shapes (circles for example) to represent a damage hole, rather than using live fire damage. Using live ammunition to cause damage on a model is not ideal due to the random nature of the damage that can ensue; this will often lead to irregular shapes that will differ from test to test, making experimental variables hard to control. Irwin ${ }^{2}$ modelled gunfire damage as a circular hole, following investigations which showed this to be the most common shape approximation of damage. This is expected from non-explosive armour-piercing shells, although even with explosive shells it was concluded that the 'Fragment Spray Zone' also passed through the wing in a roughly circular shape. Due to these findings, the modelling of damage in this work will use circular shaped holes.

Irwin identified the main flow features produced by a single simulated damage hole as a Weak Jet and a Strong Jet (see Figure 1), a third case occurs in transition between a weak and strong jet which can display features of both. It was found that battle damage reduces lift, increases drag and changes pitching moment and these effects are most significant for strong jets. If air speed is constant, three main variables determine the jet strength; damage size, damage location and incidence angle. ${ }^{7}$ The weak jet typically occurs at low incidence angles or with small holes, when there is a relatively small pressure difference between the pressure and suction surface of the wing. The strong jet case occurs at higher incidences, when the pressure difference is high between the pressure and suction surfaces, for smaller holes and at all incidence angles for very large holes.

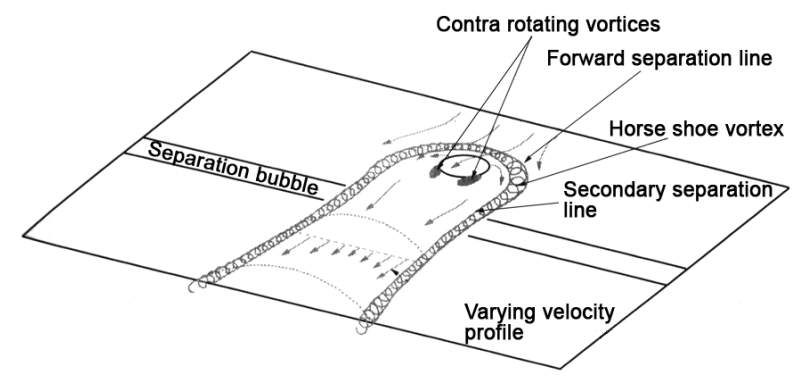

(a) Weak Jet

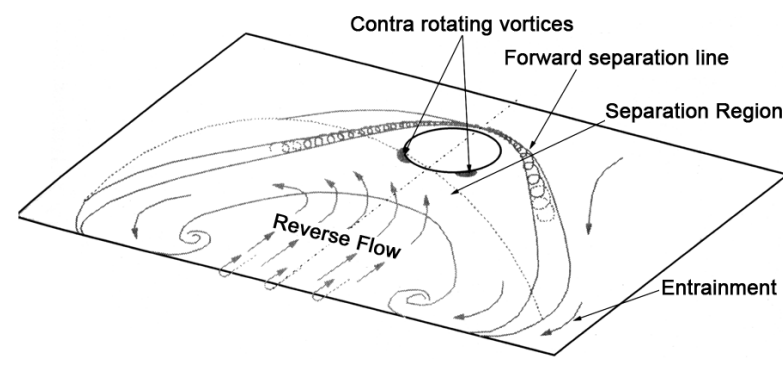

(b) Strong Jet

Figure 1: Key flow features of a Weak and Strong jet from a single simulated battle damage hole, (Irwin $\left.{ }^{2}\right)$

The weak jet is identifiable by the narrow reverse flow region and the lack of freestream penetration. The jet and the Horse-Shoe Vortex (HSV) remain very close to the upper surface of the wing up to the trailing edge and the Counter-Rotating Vortex Pair (CVP) can be seen on the circumference of the damage hole. The strong jet penetrates the freestream further and creates a larger reverse flow region in both the vertical and spanwise direction. The HSV is much larger and does not remain attached to the wing surface. The CVP can also be seen on the circumference of the damage hole, however the location has moved towards the span. Figure 2 shows the flow features of a typical flat plate JICF for comparison; the formation of the 
HSV can be seen just in front of the jet. The jet trajectory is affected by the cross-flow, bending it in the cross-flow direction and the effects of the CVP are seen in the jet. The features of JICF seen in Figure 2 are described in more detail on Page 5.

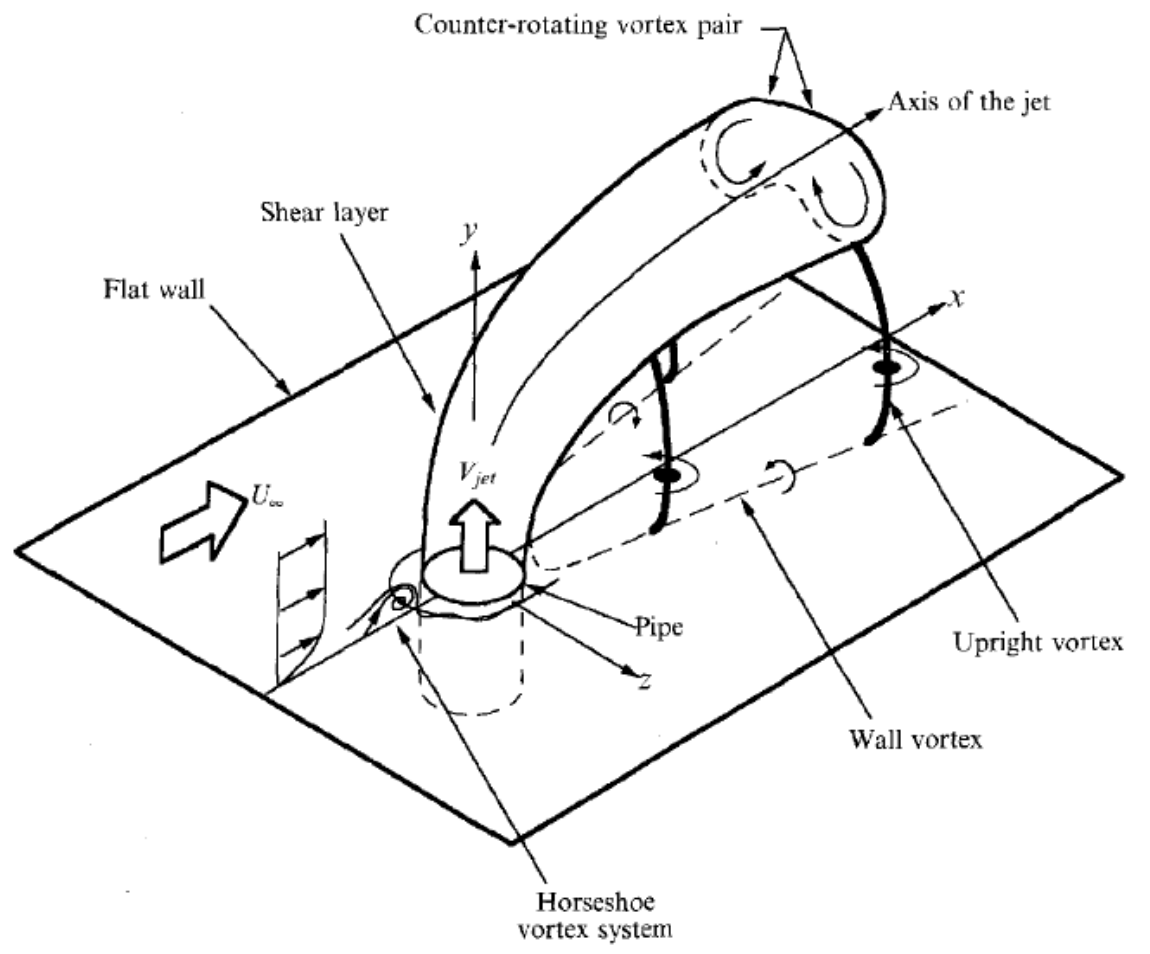

Figure 2: Vortex system from typical flat plate in cross-flow, from Kelso et al ${ }^{8}$

Previous battle damage work has concentrated on balance measurements to determine lift and drag increments with only limited flow field information from surface flow visualisation. This has provided a limited understanding of the flow field and pertinent flow mechanisms, hence the need for more in-depth studies involving PIV. CFD simulations have been done by Yang et $\mathrm{al}^{9}$ and Saeedi et al ${ }^{10}$ but have not been validated, furthering the requirement for 3-Dimensional flow field data. Even though the fundamental flow features produced by battle damage flow have been documented, they are not understood in any detail. Using PIV for the first time will help to shed light on the 3D nature of the flow, and improve understanding of the transition between weak and strong jets.

Render \& Pickhaver ${ }^{11}$ extended Irwin's studies and investigated the influence of battle damage on a NASA LS(1)-0417MOD aerofoil at a Reynolds number of 500,000. Figure 3 shows the force coefficient increment data (force coefficient of damaged wing minus force coefficient of undamaged wing) for a simulated battle damage hole of $20 \%$ chord, with both upper and lower surface holes located at mid-chord. Below the zero lift angle of $-2.5^{\circ}$ the jet flow direction was reversed and penetrated on the lower surface, leading to a reduction in negative lift. Both the lift and drag increments worsen with incidence, until $10^{\circ}$ when the trend is reversed and the increments reduce. Render and Pickhaver put this down to the wing being close to stall and having significant separation on the upper surface. However, at all incidences, battle damage caused a reduction in lift coefficient and and increase in drag coefficient.

Figure 4 shows the pressure data for the upper and lower surfaces of the damaged aerofoil with a damage hole of $20 \%$ c located at mid-chord, along with the undamaged wing data. The hole bounds are shown as vertical dashed lines at $0.4 x / c$ and $0.6 x / c$. On the upper surface there is an adverse pressure gradient in front of the hole for the centreline and $\mathrm{R}=0.5$ locations $^{\ddagger \ddagger}$. Compared to the undamaged case, the pressure coefficient $\left(C_{p}\right)$ values have decreased with the presence of the damage hole, however the adverse pressure

${ }^{\ddagger \ddagger} \mathrm{R}=0.5$ is 0.5 hole radii from the centreline of the hole, measured in the spanwise direction 


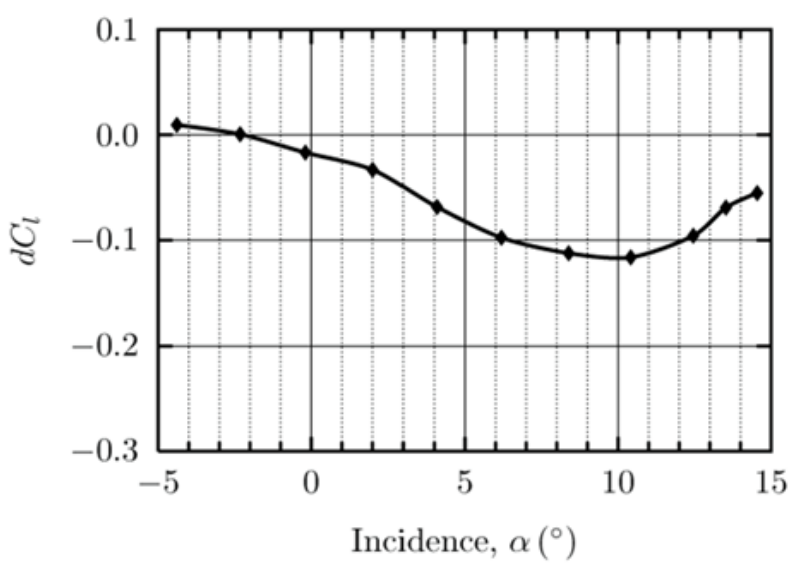

(a) Lift Coefficient Increment $d C_{l}$

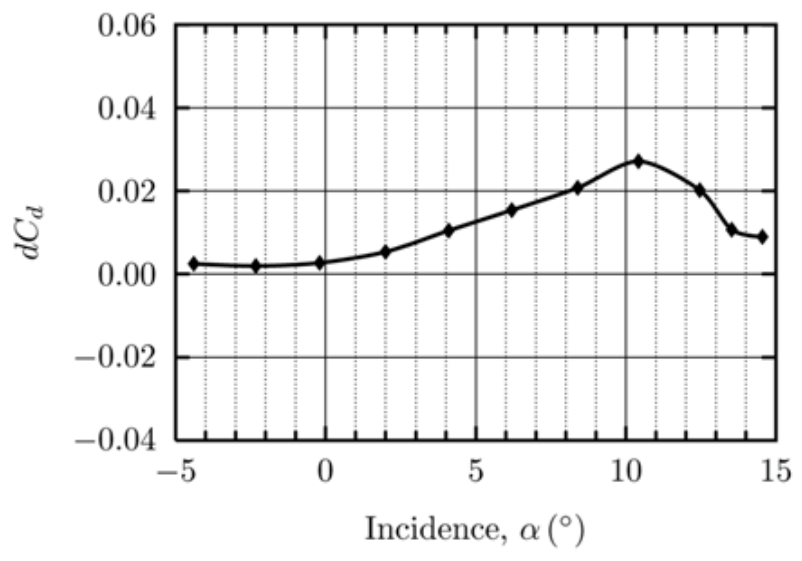

(b) Drag Coefficient Increment $d C_{d}$

Figure 3: Force increments for a damage hole of $20 \%$ c located at mid-chord, from Render and Pickhaver ${ }^{11}$

gradient has increased. The reduction in $C_{p}$ indicates a reduced velocity, but the increase in pressure gradient can be attributed to the blockage effects of the jet, as found by Doligalski et al. ${ }^{12}$ The cross-flow velocity on the surface of the wing is reduced as the flow moves around the blockage caused by the jet. The pressure gradient behind the hole is small for all spanwise locations, even though a pressure gradient was present for the undamaged wing, indicating that there is very little change in velocity in this region.

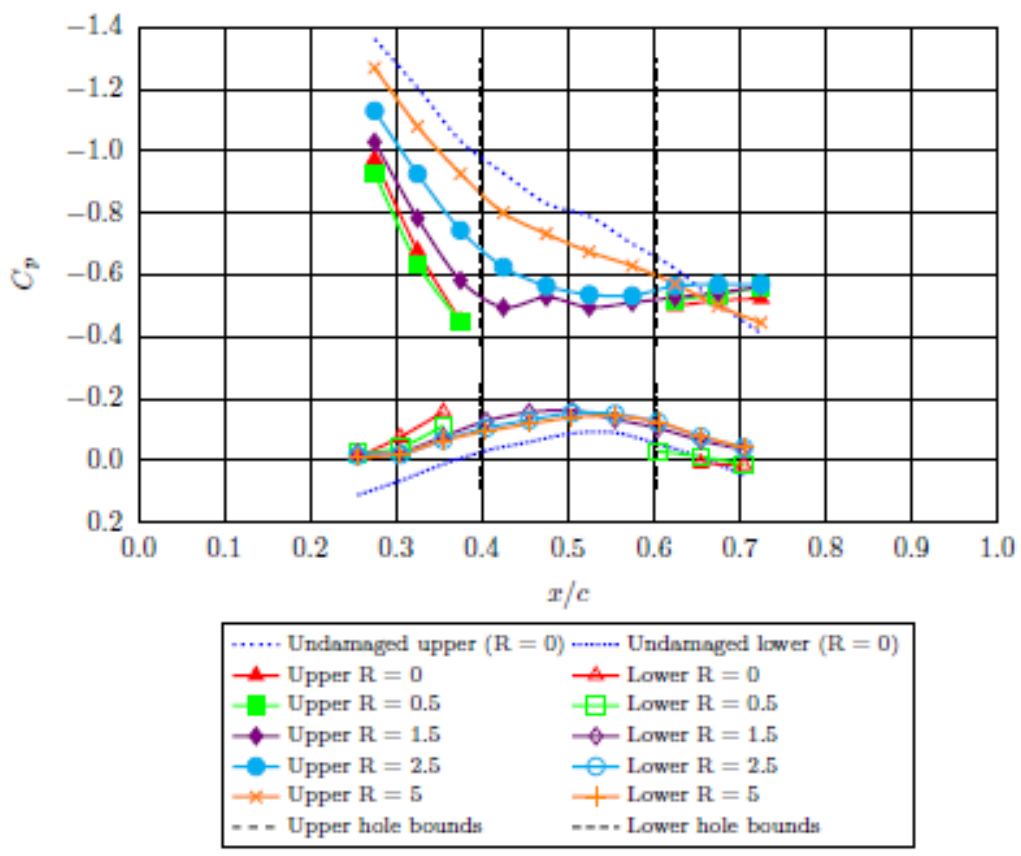

Figure 4: Pressure data for the damaged wing at $8^{\circ}$, from Render and Pickhaver ${ }^{11}$

From previous studies of battle damaged wings, it is clear that a jet of air passes through the wing and out into the freestream flow above the wing, very similar to classical jets in cross-flow. Mahesh ${ }^{13}$ defines the term 'Jet in Cross-flow' as referring to a jet of fluid that exits an orifice to interact with the surrounding fluid that is flowing across the orifice. By this simple definition the problem being described in this paper can be thought of as a JICF. Important parameters in the study of JICF are jet velocity ratio, $V_{R}$ (Equation 1 ), where $U_{j e t}$ is the mean velocity across the hole exit and $U_{\infty}$ is the freestream (or cross-flow) velocity, 
and momentum flux ratio, $J$ (Equation 2), where $\rho_{\text {jet }}$ and $\rho_{\infty}$ are the density of the jet fluid and cross-flow fluid respectively.

$$
\begin{gathered}
V_{R}=\left(U_{j e t} / U_{\infty}\right) \\
J=\rho_{j e t} U_{j e t}^{2} / \rho_{\infty} U_{\infty}^{2}
\end{gathered}
$$

In classic JICF $V_{R}$ and $J$ are much easier to calculate due to the experimental arrangements, in most cases the jet will be running full and passing through the full area of the pipe. With this setup, it can be assumed that the mass flow is the same at all points in the pipe making measurements simple. In the case of battle damage, the jet is not driven by an external source such as a pump, but exists due to the pressure difference between the pressure and suction surfaces of the aerofoil. There is also a cavity in the centre of the wing, meaning the jet enters and leaves a large space rather than passing through a solid pipe. Further, as will be seen in the results section, the jet does not exit over the full area of the hole, but just a fraction of it. This makes the calculation and definition of $V_{R}$ and $J$ difficult.

Key flow features usually seen with JICF are the horse-shoe vortices (HSV) and counter-rotating vortex pairs (CVP). These features are visible in Figure 2 along with others associated with JICF, including detachment from the surface, freestream penetration and the bending over of the jet by the cross-flow. For classic JICF opinions differ on the physics behind the formation of these features. However, there is a general agreement that they are present at high velocity ratios $(>2)$. At low velocity ratios Mahesh ${ }^{13} \mathrm{states} \mathrm{that}$ the flow structure may be fundamentally different; fluid in the near field is strongly accelerated, which affects the jet trajectory, entrainment and mixing behaviour. As will be discussed later, this is one area where JICF and battle damaged wing flows differ; the CVP appears to form at much lower velocity ratios $(<1)$.

Horse-shoe vortices form upstream of the hole leading edge, where the wing boundary layer encounters an adverse pressure gradient upstream of the jet, separates and forms span-wise vortices that move around the jet. ${ }^{13}$ The counter-rotating vortex pair (CVP) is highly unsteady and asymmetric, ${ }^{13}$ with different opinions on how they form. The general consensus is that CVP formation results from 'modification of the jet vorticity by the cross-flow ${ }^{13}$. 


\section{Experimental Setup}

The tests were conducted in the Loughborough University Low Turbulence Wind Tunnel. The wind tunnel is of open return design with a closed working section measuring $0.45 \mathrm{~m} \times 0.45 \mathrm{~m}$, typical inlet turbulence intensity is approximately $0.1 \%$. For convenience, the model was attached to an under-floor force balance, although no measurements were taken from the balance in the present study. The tests were performed at a wind speed of $37 \mathrm{~m} / \mathrm{s}$, which gave a Reynolds Number of 500,000 with a model of $200 \mathrm{~mm}$ chord. A schematic diagram of the wind tunnel facility is shown in Figure 5.

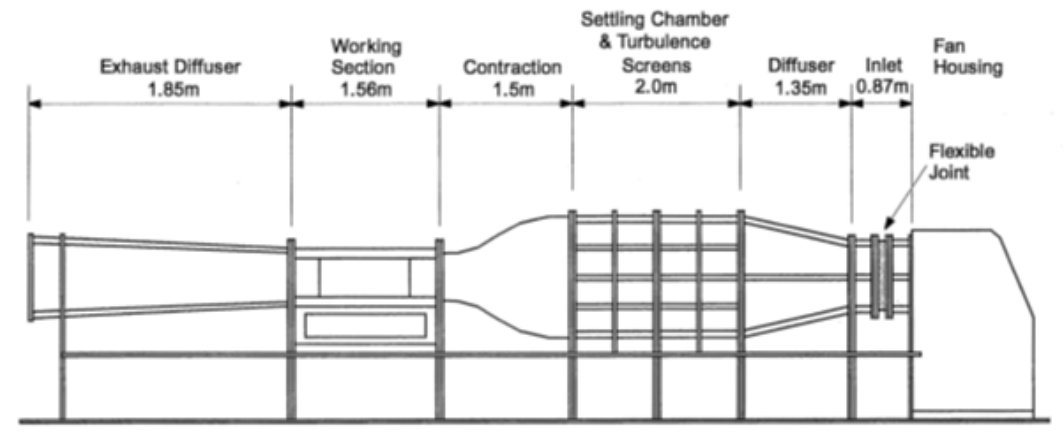

Figure 5: Schematic of Loughborough University Low Turbulence Wind Tunnel ${ }^{11}$

A single hole of $20 \%$ chord diameter located at mid-chord on the upper and lower surface of a wing was chosen for this work as it provides a typically survivable damage case that produces both a weak and a strong jet over the incidence range tested. The wing model used a NASA LS(1)-0417MOD aerofoil which was chosen because of its close similarity to existing reconnaissance UAV's. To represent typical construction of aircraft, it was of hollow construction and used removable composite skin panels. Damage was applied to the panels using a laser cutter and the panels were fixed using countersunk screws so it sat flush with the wing surface. A cutaway of the wing model is shown in Figure 6.

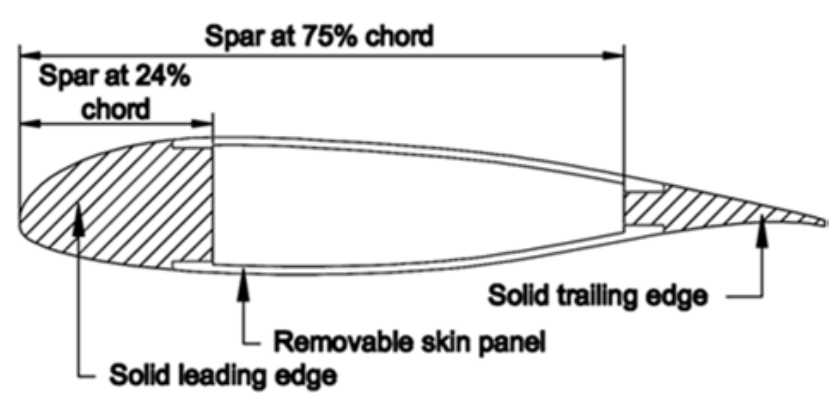

Figure 6: Model construction showing hollow cavity ${ }^{11}$

Two component PIV using LaVision equipment was used in three streamwise planes across the hole; the first was taken along the centreline of the hole, the second at a distance of $0.5 \mathrm{R}$ off centre and the third at a distance of 1R off centre. The light sheet for the PIV (shown in green in Figure 7) was produced by a 200mJ double pulsed Nd:YAG laser, located above the working section of the wind tunnel on a traverse mechanism. The laser emits a circular beam of $4 \mathrm{~mm}$ diameter which when focused through a cylindrical lens gave a sheet of approximately $1 \mathrm{~mm}$ thickness at the measurement plane. A single FlowMaster Imager Intense CCD camera with a resolution of $1376 \times 1040$ pixels was used to record the PIV images using a 105mm focal length Nikon lens with an aperture value of $1 / 2.8$. The camera and laser were controlled by an external Programmable Timing Unit (synchronizer), which sets the trigger rate at $5 \mathrm{~Hz}$, the typical time between images within a pair (interframe time or $d t$ ) was 30-40 $\mu s$; this was optimised for each setup position. The flow was seeded with 1-2 $\mu \mathrm{m}$ particles produced by a LaVision aerosol generator at 4 bar using olive oil as the seeding fluid. It was shown by Hollis ${ }^{14}$ that oil particles of $2 \mu \mathrm{m}$ faithfully followed the flow in air for accelerations greater than seen in this experiment, giving an acceptable particle velocity lag at worst case scenario. 


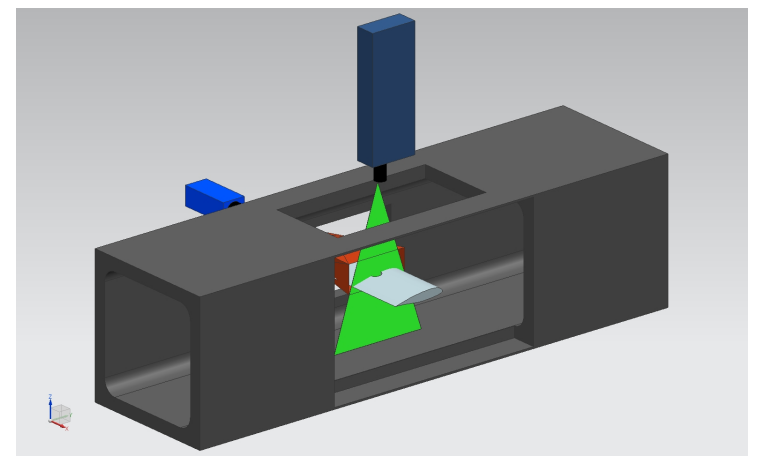

(a) Full Working Section

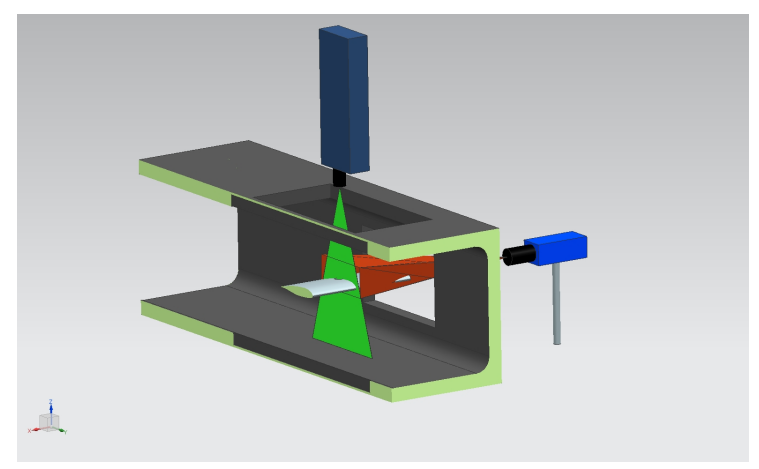

(b) Cutaway

Figure 7: PIV setup in wind tunnel showing optical access, laser sheet and camera field of view

The system was calibrated in each measurement plane using a two dimensional calibration plate. The markings on the plate represented a known dimension in $x$ and $y$, allowing the software to perform a spatial calibration for use in the velocity measurements. To verify that the PIV and wind tunnel settings were appropriate, 50 image pairs were taken over a range of $d t$ values and processed to check for data quality and peak locking. The particle image diameter was between 1 and 2 pixels (the apparent size of the particle based on the scattered light entering the camera lens) meaning pixel biasing effects were within acceptable limits in regard to the quality of the measured flow patterns and flow statistics. ${ }^{15}$ One thousand image pairs were taken for each incidence angle and processed using LaVision's DaVis 8.0 software. An additional test taking 2000 images showed no significant improvement in the statistical accuracy of the PIV data (Hollis ${ }^{14}$ ). The processing was performed using a multi-pass technique, starting with one pass at a window size of $64 \times 64$ pixels then decreasing to $32 \times 32$ for three passes. The overlap between the interrogation windows was constant at $50 \%$. The vector fields each contain $115 \times 87$ vectors, totaling 10005 data points. The parts of the image where the wing model was visible were masked to reduce the chance of spurious vectors.

The accuracy was optimised for the jet and reverse flow region, with the trade off being a less than optimal arrangement in the freestream. The average particle displacement in the reverse flow region is 7.4 pixels, conforming to the design rule defined by Adrian \& Westerweel ${ }^{15}$ shown in Equation (3) where the displacement vector $|\Delta x|$ is less than a quarter of the linear dimension of the final interrogation area, $\mathrm{L}_{\mathrm{I}}$ :

$$
|\Delta x|<\frac{1}{4} \mathrm{~L}_{\mathrm{I}}
$$

In the freestream the value is much higher at 22 pixels. Although this value is higher than the optimum the flow is nominally consistent and laminar in its transition through the area of interest, meaning the software easily calculates the velocity, helped by the multi-pass processing technique. In the freestream the instantaneous velocity fields show over $99.8 \%$ first choice vectors, higher than the turbulent reverse flow region which produces around $90 \%$. The remaining data has been removed during processing due to it being more than 2 standard deviations away from neighbouring vectors. This technique reduces spurious vectors and improves the average vector field.

The flow visualisation photographs taken from Pickhaver ${ }^{7}$ are used during the analysis of the results and these were obtained using a mixture of titanium dioxide powder, linseed oil and paraffin. The mixture was applied to the upper surface of the model using spanwise strokes of a paint brush. The tunnel was then run at normal test speed $(37 \mathrm{~m} / \mathrm{s})$ and the flow patterns photographed from above the working section. The wind tunnel was left running during this process to ensure the visible flow patterns were those caused by the airflow and not from gravity after the airflow was turned off. 


\section{Results \& Analysis}

\section{A. Flow Field}

Figure 8 shows the centreline PIV images and surface flow visualisation photographs for incidence angles of $2^{\circ}$ and $8^{\circ}$. The PIV images display velocity magnitude divided by freestream velocity $\left(V / V_{\infty}\right)$; the values peak at 1.35 due to the combination of accelerated flow on the suction surface of the wing and the increased blockage effect of the jet at the higher incidence angles increasing the flow speed around the wing model. The data shows that the hole (indicated by the two parallel lines normal to the chord) produces a jet that penetrates the free stream in both cases. The freestream penetration is greater for the higher incidence angle and the reverse flow region (in blue) has grown significantly (see Figure 9 for more on the reverse flow region). The maximum freestream penetration is around $2 \mathrm{R}$ for the strong jet case, as opposed to $1 \mathrm{R}$ for the weak jet, measured from the wing surface.

Comparing the two incidence angles, the 2 degree case has less effect in the spanwise direction, as seen in the flow visualisation photographs. There is little or no freestream penetration over the front section of the hole due to the HSV sitting over this area. As stated in Section I, the HSV typically forms ahead of the jet, and hence the hole, however as the jet is only exiting the rear portion of the hole, formation of the HSV occurs further back. The adverse pressure gradient exists in front of the hole, as seen in Figure 4, but will continue until the start of the jet which is $15 \%-20 \%$ back from the hole leading edge $(0.15-0.2(X / D))$. At $8^{\circ}$, the spanwise effect of the jet is larger; the HSV, wake and reverse flow region are between $4 \mathrm{R} \& 5 \mathrm{R}$ either side of the hole centre, as opposed to 1.5R for the weak jet. The HSV forms in front of the hole as expected from JICF, but some part of the vortex still sits over the front of the hole.
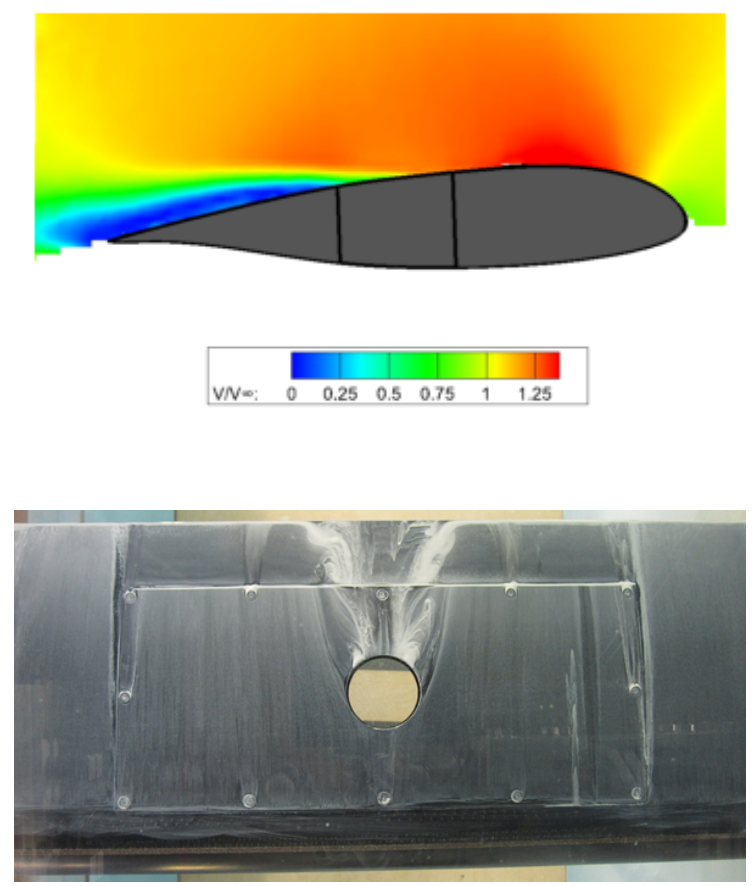

(a) 2 Degrees
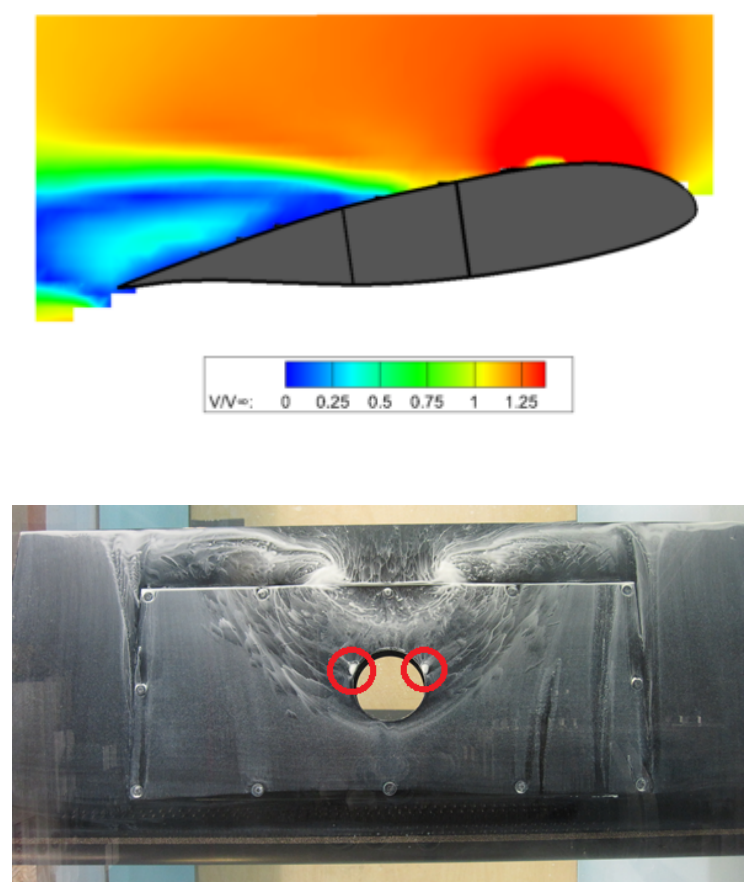

(b) 8 Degrees

Figure 8: Comparison of centreline PIV data with surface flow visualisation ${ }^{7}$

The counter-rotating vortex pair are present at both incidence angles, however the position on the hole circumference is not the same. The CVP can be seen on the circumference of the damage hole as small collections of flow visualisation fluid, indicated by the red circles in Figure $8 \mathrm{~b}$. At $8^{\circ}$ the CVP is further out toward the span and closer to the leading edge than at $2^{\circ}$. The CVP appears larger in the surface flow visualisation photographs at the lower incidence angle; this is because it is closer to the wing surface and 
has more impact on the flow visualisation fluid than at higher incidence angles when it is separated from the surface.

Figure 9 shows the downstream flow structure behind the hole for the same incidence angles $\left(2^{\circ} \& 8^{\circ}\right)$. The centre of the reverse flow structures are in a very similar position for both weak and strong jet cases, just in front of the wing trailing edge. However, the vortex centre is located further from the surface for the strong jet case providing a large space between the wing surface and the vortex centre for flow in the upstream direction.
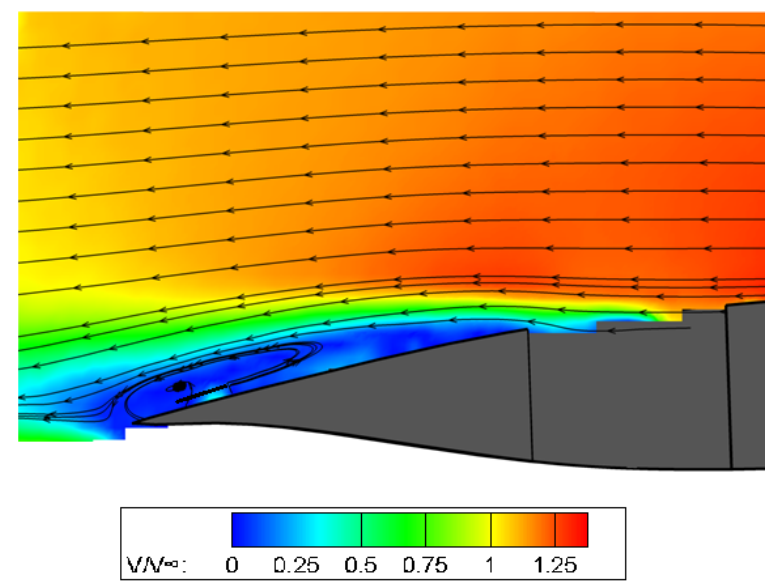

(a) 2 Degrees

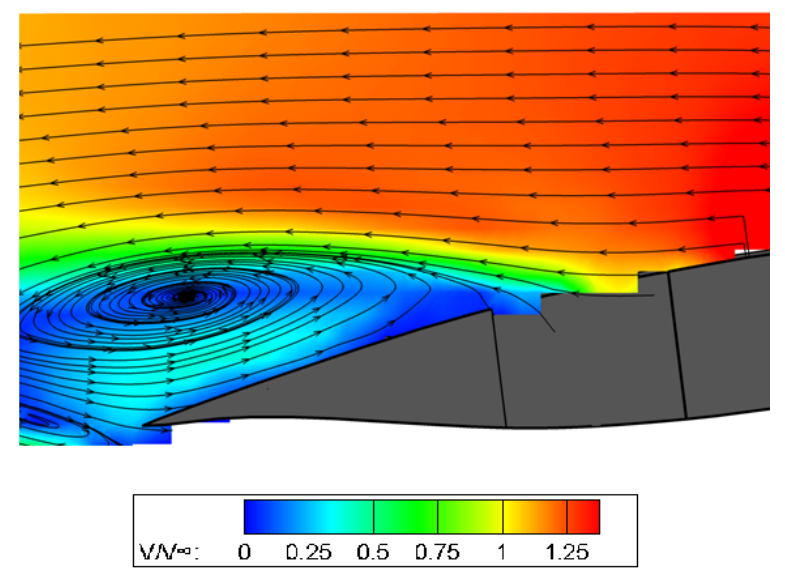

(b) 8 Degrees

Figure 9: Flow pattern behind the hole for weak and strong jet on the centreline plane

Figure 10 shows the jet flow exiting at the $0.5 \mathrm{R}$ off centre location. The $2^{\circ}$ case shows very little difference to the flow on the centreline, with changes in freestream penetration being negligible. The $8^{\circ}$ case has little difference over the front three quarters of the hole, also showing a similar flow pattern and penetration into the freestream as compared to the centreline. However, in the rear part of the hole the reverse flow is not impinging on the jet. The reduced amount of reverse flow impingement on the jet is attributed to the three dimensional nature of the flow; as the reverse flow approaches the back of the jet it not only moves up and back, but is pushed spanwise. This reduces the affect of the reverse flow on the jet away from the centre, with this effect being greater as distance from the centreline increases. 

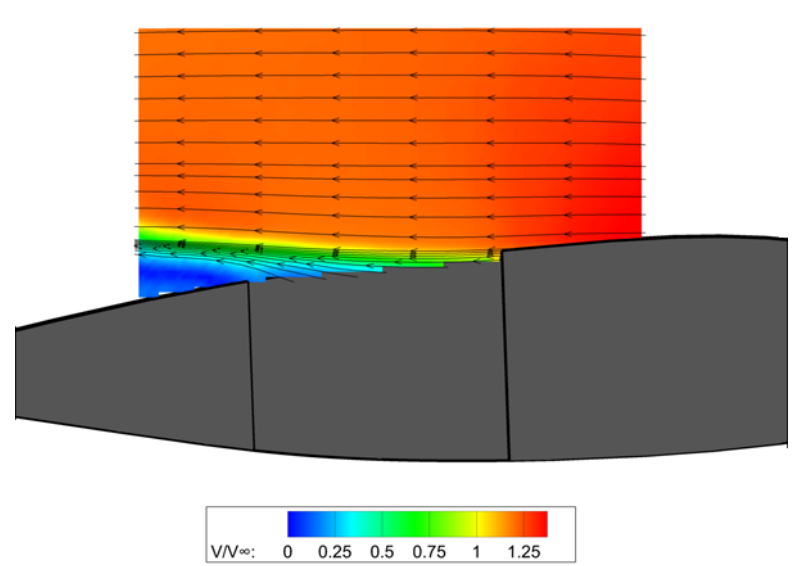

(a) 2 Degrees

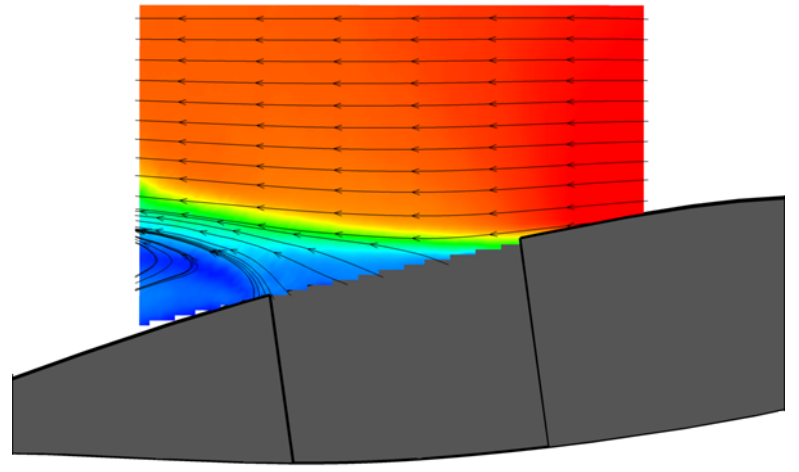

$\begin{array}{lllllll}V N \infty & 0 & 0.25 & 0.5 & 0.75 & 1 & 1.25\end{array}$

(b) 8 Degrees

Figure 10: Hole exit at $0.5 \mathrm{R}$ off centre

Moving to $1 \mathrm{R}$ off centre, at an incidence angle of 2 degrees, the reverse flow region is no wider than the width of the hole, this can be seen in the flow visualisation photograph (Figure 8a) and also in the PIV data shown in Figure 11a. This data, taken on the edge of the damage hole, shows that behind the hole the flow is traveling in the freestream direction. At $8^{\circ}$ the reverse flow region is well defined but is not preceded by an exiting jet; the bounds of the reverse flow at this location are the horse-shoe vortex and the jet shear layer.

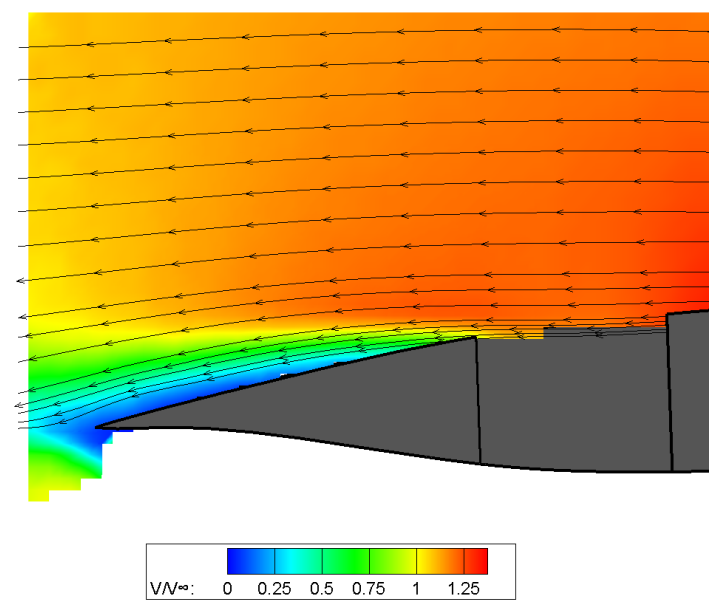

(a) 2 Degrees

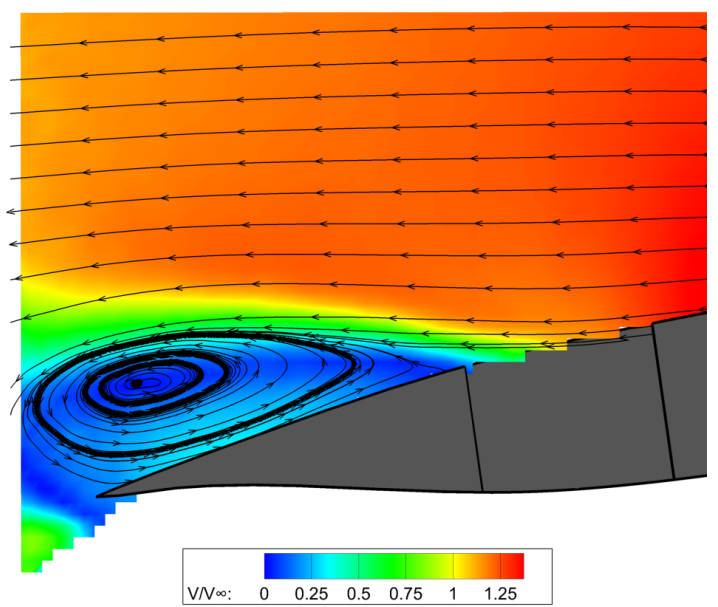

(b) 8 Degrees

Figure 11: Flow pattern behind the hole for weak and strong jet 1R off centre (on edge of hole)

Moving in a spanwise direction, the reverse flow region behind the damage hole reduces in height from a maximum at centreline $\left(2 \mathrm{R}\right.$ at $\left.8^{\circ}\right)$, to approximately $1.5 \mathrm{R}$ at $8^{\circ}$ at 1 radii off centre, ie. on the edge of the hole. This will continue to decrease to 0 at approximately $5 \mathrm{R}$ away from the hole centre. 


\section{B. Velocity Ratio}

As stated in section I, defining velocity ratio can be challenging for a battle damage case. The data shows the jet exiting only a portion of the hole towards the rear. Figure 12e shows the velocity ratio across the centreline. This has been calculated by recording the velocity in the $y$ direction (normal to freestream) at 9 locations over the hole, then dividing by the tunnel freestream velocity. The $x$ component of velocity is very high over the first half of the hole (due to the cross-flow boundary layer) so it was therefore unrealistic to use the velocity magnitude; this would produce unreasonably high values of $V_{R}$ in areas with little or no freestream penetration. All incidence angles from $0^{\circ}-10^{\circ}$ were recorded but only 4 key incidence angles are displayed here.

The velocity ratio is negative at the leading edge of the hole for all incidence angles, suggesting the jet is being affected by the horse-shoe vortex sitting in front or over this area. The velocity ratio then rises at around $0.12(X / D)$ from the leading edge and plateaus up to $0.7-0.8(X / D)$. The velocity ratio across this plateau increases, as expected, with incidence angle. Beyond $0.8(X / D)$ the velocity ratio begins to vary much more with both location and incidence. For the lowest incidence angle the peak $V_{R}$ is seen at the rear of the hole. This agrees with Figure $12 \mathrm{a}$ which shows at $0^{\circ}$ there is no reverse flow impingement on the rear of the jet, this is a weak jet. At higher incidence angles the peak $V_{R}$ is seen to increase significantly and shift slightly forward due to reverse flow impingement, this is a strong jet. However, values of $V_{R}$ are still much lower than expected from JICF. The two cases that stand out are $2^{\circ}$ and $3^{\circ}$ as they fit neither the weak jet trend or the strong jet trend. These have been identified as the transitional incidence angles where the jet may exhibit features of both the weak and strong jet. Figures $12 \mathrm{~b} \& 12 \mathrm{c}$ show the time averaged PIV data for these two cases; at $2^{\circ}$ the jet appears to leave the back of the hole much like the weak jet does but the reverse flow region is clearly having an effect. At $3^{\circ}$ the reverse flow is starting to impinge on the jet and effect its trajectory, stopping it from spilling out the back of the hole. The instantaneous vector fields show that across the 1000 images, there are points in time where the transitional cases show strong jet flow features such as reverse flow impingement, and at other times behave more like a weak jet with the jet spilling out the back of the hole.

Figure 13 is a plot of peak velocity ratio against incidence, taken on the centreline. Between $0^{\circ}$ and $1^{\circ}$ the peak $V_{R}$ decreases. The flow here has a very small $y$ velocity and the jet has very little freestream penetration. The jet trajectory just above the hole is immediately skewed in the direction of the crossflow, producing a variability of the $y$ velocity in this incidence region. The steepest gradient occurs during the transitional period, identified at between $2^{\circ}$ and $4^{\circ}$, indicating that this is the region where the biggest changes are occurring. During these incidence angles the reverse flow is starting to affect the jet trajectory, where the jet is not swept back immediately by the cross-flow but penetrates more vertically, accounting for the sharp increase in $y$ velocity. Above $4^{\circ}$ the jet is strong and the $V_{R}$ increases consistently with incidence. 


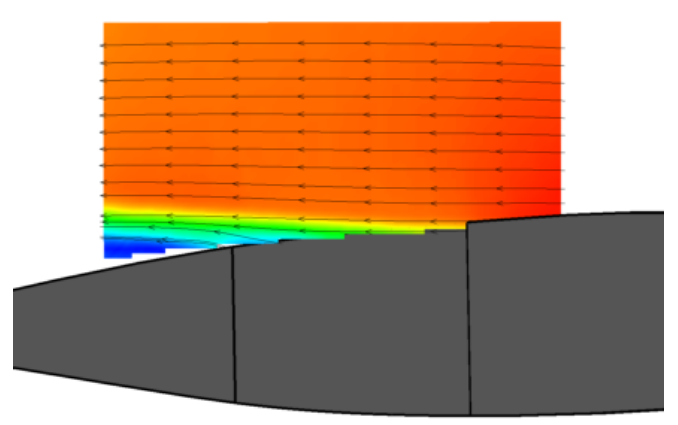

\begin{tabular}{llllll|l|l|}
\hline & & & & & & & \\
\hline VNo: & 0 & 0.25 & 0.5 & 0.75 & 1 & 1.25 \\
\hline
\end{tabular}

(a) 0 Degrees

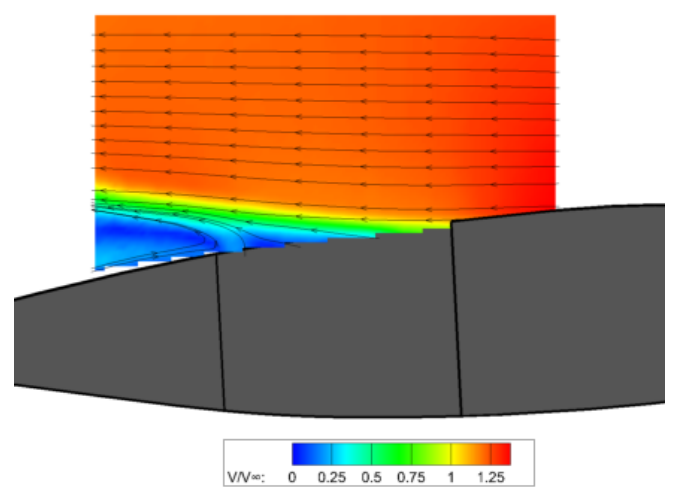

(c) 3 Degrees

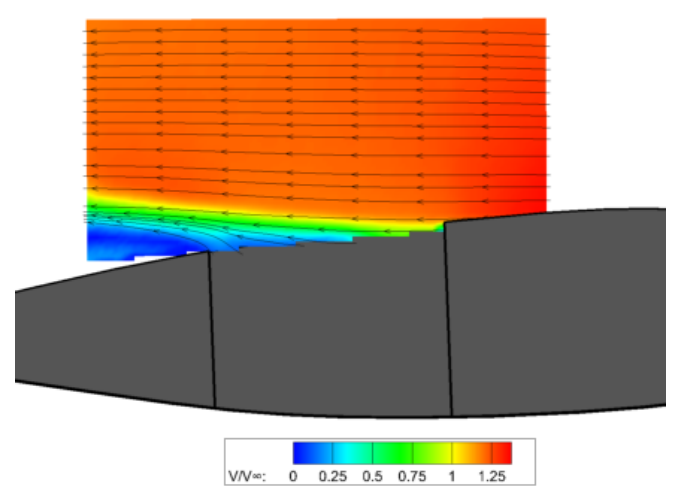

(b) 2 Degrees

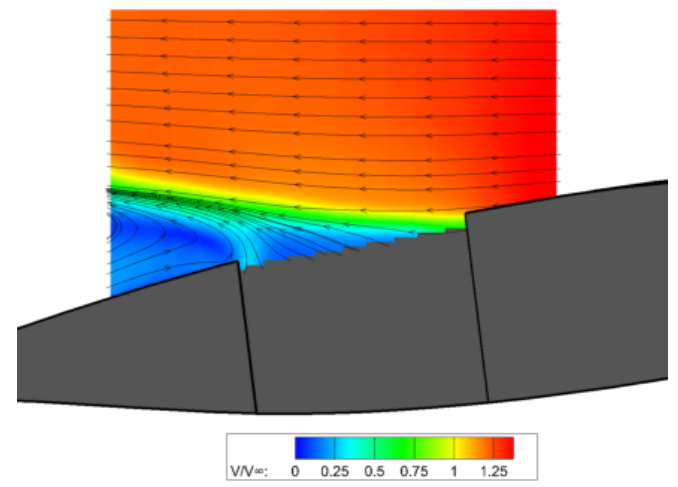

(d) 8 Degrees

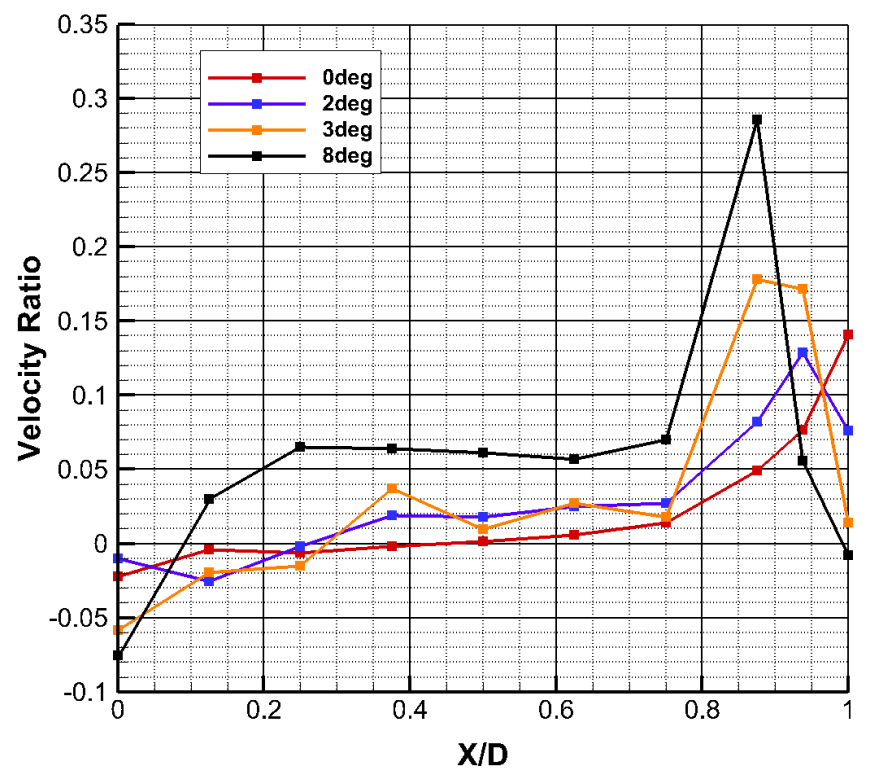

(e) Velocity Ratio corresponding to above incidence angles

Figure 12: Hole exit on centreline showing weak jet, transitional jets and strong jet cases

$$
12 \text { of } 15
$$




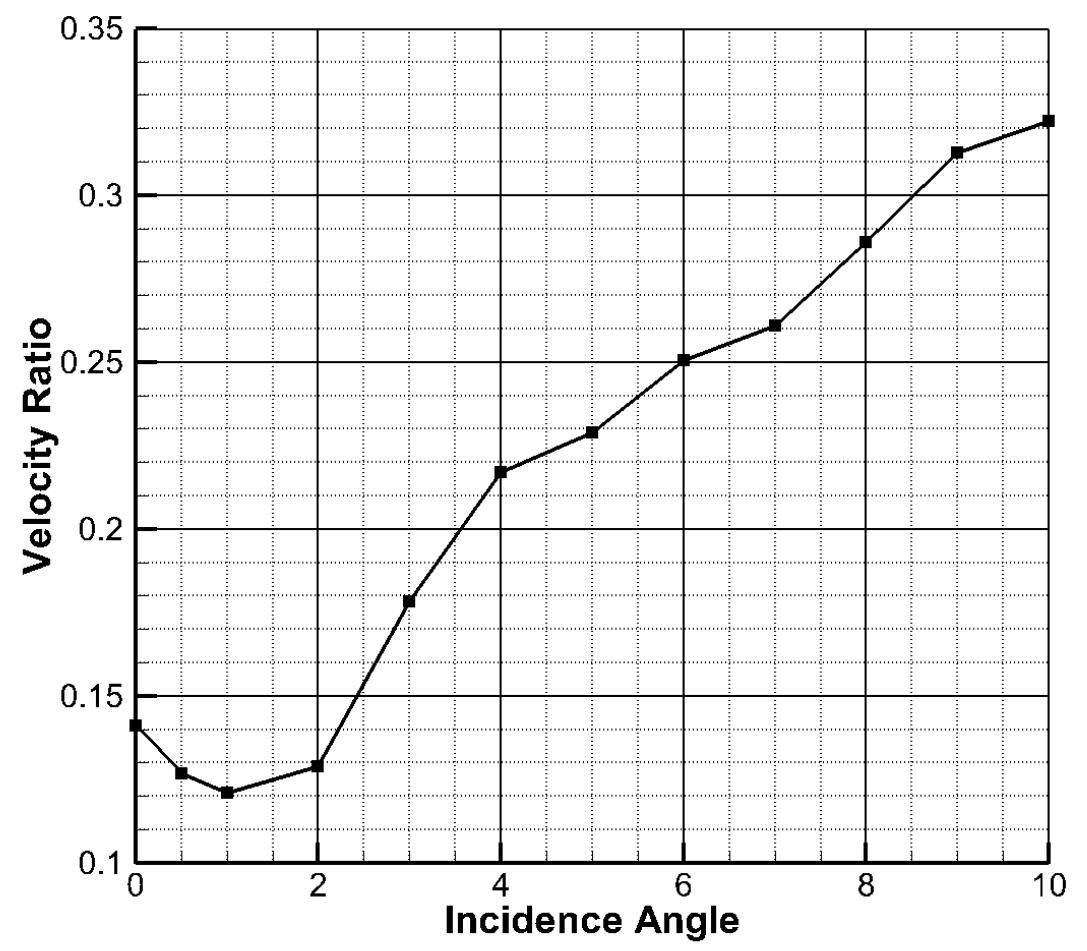

Figure 13: Peak velocity ratio with incidence

Due to the location of the CVP, taking $V_{R}$ measurements at the $0.5 \mathrm{R}$ off centre plane could be unreliable. It was discussed previously that the CVP moves as incidence changes; this movement meant that it crosses the $0.5 \mathrm{R}$ plane and affected the $V_{R}$ values towards the back of the hole. Figure 14 shows the velocity ratio, for the same incidence angles as Figure 12e, taken $0.5 \mathrm{R}$ off centre. The weak jet case at $0^{\circ}$ is negative over the majority of the hole because of the HSV sitting over the front portion, until increasing to a maximum at around $0.85(X / D)$. The trend is not the same as the centreline, where the jet was seen to spill out the back of the hole. This is due to the interaction of the CVP and the shape change of the jet created by the cross-flow, as seen by Kelso et al. ${ }^{8}$ The strong jet case $\left(8^{\circ}\right)$ shows a similar trend as centreline, holding a steady $V_{R}$ for the majority of the hole before peaking just after $0.8(X / D)$ hole diameter. The peak value is roughly half of the value at the centreline; it is not yet known whether this is a true value or if it is low due to the CVP at this location. 


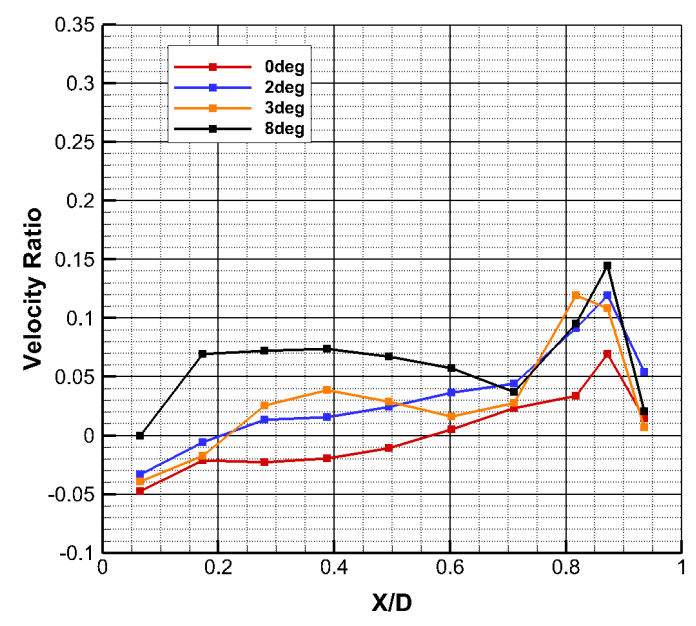

Figure 14: Velocity ratio for the off centre plane at $0.5 \mathrm{R}$

\section{Velocity Ratio and Aerodynamic Performance}

By taking the velocity ratio measurements obtained from the PIV data and comparing them to the force coefficient increments found in Figure 3, it can be seen that a relationship exists between increasing velocity ratio and increasing aerodynamic losses. Figure 15 shows $V_{R}$ plotted against both lift and drag coefficient increments, the numbers are the incidence angles corresponding to each data point. Once a transitional jet has been established, the graphs show that that lift coefficient increment decreases and drag coefficient increment increases as the velocity ratio increases. The trend is almost linear once the jet has reached the strong stage.

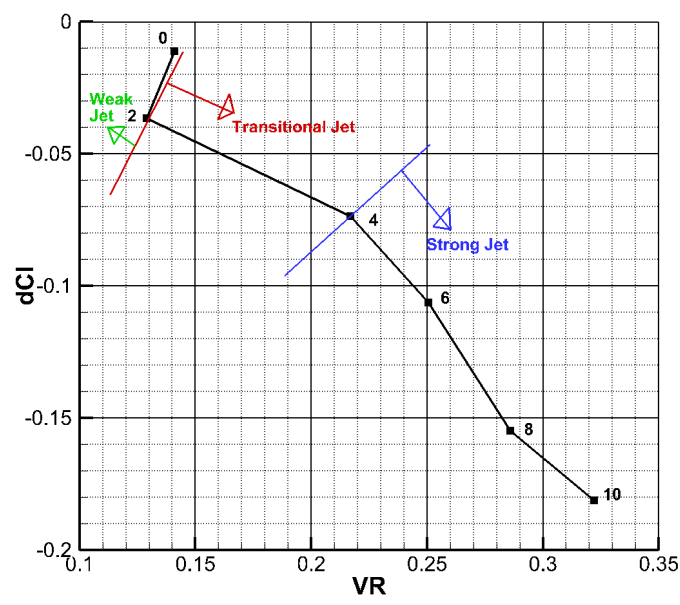

(a) $d C_{l}$

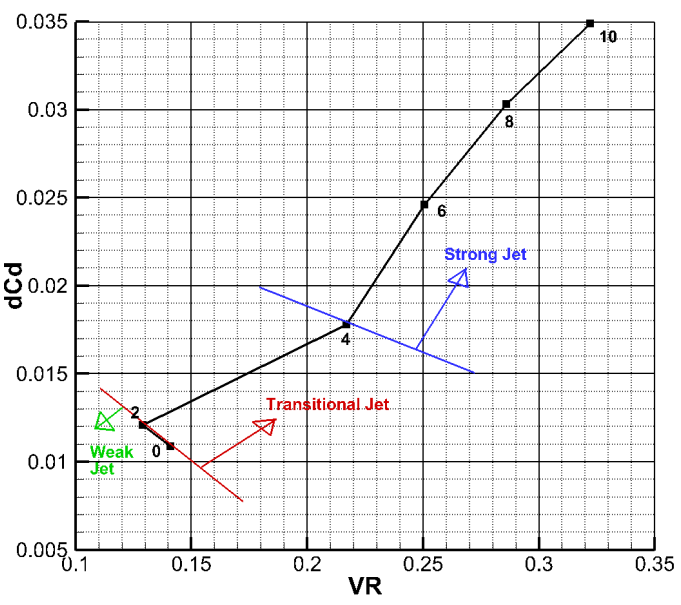

(b) $d C_{d}$

Figure 15: Variation of force coefficient increments with velocity ratio 


\section{Conclusion}

Particle Image Velocimetry has been shown to provide reliable and consistent results which provide good agreement with previous surface flow visualisation tests on battle damaged aerofoils.

Large variations in the flow structure were observed over the range of incidences tested as the jet transitioned from weak at low incidence to strong at higher incidences. When a weak jet was present, the through flow spilled out of the back of the hole. With a strong jet, the reverse flow downstream of the hole impinged on the back of the jet producing a more vertical trajectory and greater freestream penetration.

The battle damage jet never fully occupied the hole and the jet velocity profile was highly skewed towards the rear of the hole. Measured peak velocity ratios were significantly lower than those found for Jets In Cross-Flow (JICF). Weak jet behaviour was observed for battle damage at velocity ratios as low as 0.12 and strong jet behaviour at velocity ratios as low as 0.22 . JICF studies indicate that a much higher ratio $(>2)$ is required to establish a strong jet.

Once the strong jet was established there was an almost linear decrease in lift coefficient with increase in velocity ratio, whilst drag coefficient increased in an almost linear manner with increasing velocity ratio. At the highest incidence tested $\left(10^{\circ}\right)$ the peak velocity ratio of 0.32 resulted in a reduction of the lift coefficient by 0.18 and an increase in the drag coefficient of 0.035 .

\section{Acknowledgments}

Thanks go to David Cooper for the technical support he provided during the experiments and to AnnaKristina Perry for her help with experimental setup and laser alignment.

\section{References}

${ }^{1}$ Clyde Hayes. Effects of simulated wing damage on the aerodynamic characteristics of a swept wing model. Technical Report NASA TM X-1550, NASA, 1968.

${ }^{2}$ Andrew J. Irwin. Investigation into the aerodynamic effects of simulated battle damage to a wing, loughborough university thesis, 1999.

${ }^{3}$ M. Mani and P. M. Render. Experimental investigation into the aerodynamic characteristics of aerofoils with triangular and star shaped through damage. In 23rd AIAA Applied Aerodynamics Conference, Toronto, Ontario, Canada,(AIAA-20054978), 2005.

${ }^{4}$ J. G. Robinson, K. W: Leishman. Effects of ballistic damage on the aerodynamics of helicopter rotor airfoils. Journal of Aircraft, 35(5):695-695-703.

${ }^{5} \mathrm{M}$. Samaad-Suhaeb. Aerodynamics of battle damaged finite aspect ratio wings, loughborough university thesis, 2008.

${ }^{6} \mathrm{~S}$ : Ouibrahim Djellal A. Aerodynamic performances of battle-damaged and repaired wings of an aircraft model. Journal of Aircraft, 45(6):2009-2009-2023, 2008.

${ }^{7}$ T. W. Pickhaver. Prediction and validation of the aerodynamic effect of simulated battle damage on aircraft wings, loughborough university thesis, 2014.

${ }^{8}$ R. M. Kelso, T. T. Lim, and A. E. Perry. An experimental study of round jets in crossflow. Journal of fluid mechanics, 306:111-144, 1996.

${ }^{9}$ Z. Yang, M. Samaad-Suhaeb, and P. M. Render. Computational study of a battle damaged finite aspect ratio wing. In 30th Applied Aerodynamics Conference, 2012.

${ }^{10} \mathrm{M}$. Saeedi, F. Ajalli, and M. Mani. A comprehensive numerical study of battle damage and repairs upon the aerodynamic characteristics of an aerofoil. The Aeronautical Journal, 114(1158):469-483, 2010.

${ }^{11} \mathrm{P} . \mathrm{M}$. Render and T. W. Pickhaver. The influence of hole orientation on the aerodynamics of battle damaged wings. In 30th AIAA Applied Aerodynamics Conference, 2012.

${ }^{12}$ T. L. Doligalski, C. R. Smith, and J. D. A. Walker. Vortex interactions with walls. Annual Review Fluid Mechanics, 26:573-616, 1994.

${ }^{13}$ Krishnan Mahesh. The interaction of jets with crossflow. Annual Review Fluid Mechanics 2013, 2013.

${ }^{14}$ D. Hollis. Particle image velocimetry in gas turbine combustor flow fields, loughborough university thesis., 2004.

${ }^{15}$ R. J. Adrian and J. Westerweel. Particle Image Velocimetry. Cambridge University Press, New York, 2011. 